Research Paper

\title{
The Predictive Value of Albumin-to-Alkaline Phosphatase Ratio for Overall Survival of Hepatocellular Carcinoma Patients Treated with Trans-Catheter Arterial Chemoembolization Therapy
}

\author{
Zhan-Hong Chen ${ }^{1,2^{*}}$, Xiao-Ping Zhang $3^{3 *}$, Xiu-Rong Cai ${ }^{*}$, Si-Dong Xie ${ }^{4}$, Meng-Meng Liu ${ }^{1}$, Jin-Xiang Lin ${ }^{1}$,

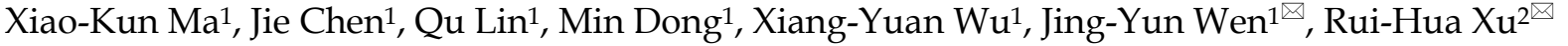 \\ 1. Department of Medical Oncology and Guangdong Key Laboratory of Liver Disease, Third Affiliated Hospital of Sun Yat-sen University, 600 Tianhe Road, \\ Guangzhou, 510630, China \\ 2. Department of Medical Oncology of Sun Yat-sen University Cancer Center, State Key Laboratory of Oncology in South China, Collaborative Innovation \\ Center for Cancer Medicine, 651 Dongfengdong Road, Guangzhou, 510060, China \\ 3. Meihua Street Community Health Service Center, Yuexiu District Guangzhou, 510000, China \\ 4. Department of Radiology and Guangdong Key Laboratory of Liver Disease, Third Affiliated Hospital of Sun Yat-sen University, 600 Tianhe Road, \\ Guangzhou, 510630, China \\ * These authors contributed equally to this work
}

$\triangle$ Corresponding authors: Jing-Yun Wen: Address: Department of Medical Oncology and Guangdong Key Laboratory of Liver Disease, Third Affiliated Hospital of Sun Yat-sen University, 600 Tianhe Road, Guangzhou 510630, People's Republic of China; Tel.: +86-20-85252212; E-mail: wenjingy@mail.sysu.edu.cn and Rui-Hua Xu: Address: Department of Medical Oncology of Sun Yat-sen University Cancer Center, State Key Laboratory of Oncology in South China, Collaborative Innovation Center for Cancer Medicine, 651 Dongfengdong Road, Guangzhou 510060, China; Tel.: 86-20-87343795; Email: xurh@sysucc.org.cn

(1) Ivyspring International Publisher. This is an open access article distributed under the terms of the Creative Commons Attribution (CC BY-NC) license (https://creativecommons.org/licenses/by-nc/4.0/). See http://ivyspring.com/terms for full terms and conditions.

Received: 2018.03.16; Accepted: 2018.08.15; Published: 2018.09.08

\begin{abstract}
Background: We have previously reported the prognostic value of the albumin-to-alkaline phosphatase ratio (AAPR) for advanced hepatocellular carcinoma $(\mathrm{HCC})$ patients who are not receiving any standard anticancer therapy. However, the prognostic value of the AAPR for HCC patients treated with trans-catheter arterial chemoembolization therapy (TACE) was not investigated.

Methods: We retrospectively analysed $372 \mathrm{HCC}$ patients treated with TACE (the training cohort) and applied receiver operating characteristic curves (ROC curves) to identify the best cut-off value for the AAPR in this cohort. Then, univariate analyses by the Kaplan-Meier method and multivariate analysis by a Cox proportional hazards regression model were conducted. Both comparisons of the ROC curves and the likelihood ratio test (LRT) were employed to evaluate the abilities of different factors in predicting the survival of patients in this cohort. Finally, the prognostic value of the AAPR was validated in two cohorts: one included $202 \mathrm{HCC}$ patients treated with supportive care (validation cohort I), and the other included $82 \mathrm{HCC}$ patients treated with TACE (validation cohort II).

Results: We identified 0.439 as the best cut-off value of the AAPR by ROC curve analysis. An AAPR > 0.439 was significantly correlated with a lower frequency of Child-Pugh grade B, portal vein tumour thrombus (PVTT), T3-4 and lymph node metastasis $(P<0.05)$. The median overall survival $(O S)$ of the patients with an AAPR $>0.439$ was significantly longer than that of those with an AAPR $\leq 0.439(58.4 \mathrm{~m}$ vs $17.8 \mathrm{~m}$, respectively, $P<0.001)$. The AAPR was identified as an independent prognostic factor after univariate and multivariate analyses $(H R=0.636, P=0.003)$. The independent prognostic value of the AAPR was also confirmed in validation cohorts I and II. Additionally, we substituted the AAPR for the Child-Pugh grade in the CLIP system and integrated the AAPR into the TNM system. We found that the area under the curve (AUC) of the AAPR-CLIP system was significantly larger than that of the CLIP and the TNM when predicting 3-month, 6-month, 1-year and 2-year survival $(P<0.05)$. There was no significant difference between the AUCs for the AAPR-CLIP and the AAPR-TNM. The LRT suggested that both AAPR-CLIP and AAPR-TNM had significantly larger $X 2$ values and smaller AIC values than that of their corresponding primary system $(P<0.05)$.
\end{abstract}


Conclusions: The AAPR was an independent prognostic index for the HCC patients treated with TACE. Both AAPR-CLIP and AAPR-TNM outperformed their corresponding primary system in predicting $O S$ in the current study.

Key words: albumin-to-alkaline phosphatase ratio; trans-catheter arterial chemoembolization therapy; hepatocellular carcinoma; serum biomarker; prognostic factor

\section{Introduction}

Hepatocellular carcinoma (HCC) is a deadly cancer with high incidence and mortality rates [1, 2]. HCC patients in the early stages can be cured by surgery and liver transplantation. Unfortunately, many of them are diagnosed at intermediate or advanced stages due to its latent onset and lack of specific symptoms. The main treatments for these patients include TACE, sorafenib and immune checkpoint inhibitors [3-5]. The median overall survival (OS) of HCC patients treated with TACE varied from 14 to 45 months, while the median OS of those treated with sorafenib varied from 6.5 to 10.7 months [6-8]. The clinical outcomes of these HCC patients are heterogeneous because they have different tumour burdens, liver function, performance status and treatments. Several serum biomarkers related to liver function have been reported to be prognostic for HCC patients, such as alkaline phosphatase (ALP), albumin (ALB) and the albumin-to-alkaline phosphatase ratio (AAPR) [9-15].

The AAPR is a ratio of serum ALB level divided by serum ALP level. The AAPR is a novel prognostic index for $\mathrm{OS}$ in both nasopharyngeal carcinoma (NPC) and HCC. Nie M et al. reported that the AAPR might be a novel prognostic factor in patients with metastatic nasopharyngeal carcinoma after receiving cisplatin-based regimens [14]. To the best of our knowledge, only two studies have ever explored the prognostic value of AAPR in HCC patients. Chan AW et al. confirmed the AAPR was an independent prognostic factor for HCC patients receiving surgery and palliative therapy [11]. Our team has identified the AAPR as an independent factor for OS in advanced HCC patients who are not receiving any standard anticancer therapy [15]. However, no one has ever discussed the prognostic value of the AAPR for HCC patients who have received TACE treatments.

Researchers have developed more than twelve staging systems for either predicting survival or for guiding the selection of treatment regimens for HCC patients. The Barcelona Clinic Liver Cancer (BCLC) system, the Cancer of the Liver Italian Program (CLIP) score and the American Joint Committee on Cancer (AJCC) TNM staging systems are the three commonly used staging systems. The BCLC and the CLIP take both tumour burden and liver function into account, while the TNM only takes tumour burden into account [16]. Integrating a liver function index into the TNM system can improve its prognostic value. Harimoto $\mathrm{N}$ et al. established the ALBI-TNM staging system by integrating the albumin-bilirubin (ALBI) grade into the TNM and found that the ALBI-TNM score was predictive of worse recurrence-free survival [17]. Replacing the Child-Pugh grade with a new liver function index/grade is also a common way to build a new staging system and to improve its prognostic ability. A previous study substituted the ALBI grade for the Child-Pugh grade in the CLIP system and evaluated the prognostic ability of eleven staging systems among 1973 patients with HCC. The authors concluded that modification of the CLIP scoring with the ALBI (ALBI-CLIP) retained and might have improved its prognosis prediction for advanced HCC [18]. However, no one has discussed the prognostic value of the AAPR-TNM and the AAPR-CLIP for HCC patients treated with TACE.

In our current study, we investigated for the first time the prognostic value of the AAPR among 372 HCC patients treated with TACE and compared the prognostic abilities of the AAPR-TNM, AAPR-CLIP, CLIP and TNM staging systems for predicting overall survival.

\section{Methods}

\section{Patient selection and evaluation}

The training cohort recruited patients who were diagnosed with HCC and treated with TACE at the Third Affiliated Hospital of Sun Yat-sen University from July 2009 to July 2013. The validation cohort I recruited 202 HCC patients treated with supportive care at the Third Affiliated Hospital of Sun Yat-sen University from July 2009 to July 2013, and the validation cohort II recruited $82 \mathrm{HCC}$ patients treated with TACE from August 2013 to August 2014.

The training cohort and the validation cohort II included patients who met the following criteria: (1) pathologically confirmed HCC or HCC confirmed by radiological criteria from the American Association for the Study of Liver Diseases and (2) primary treatments were TACE. We excluded patients who met the following criteria: (1) patients who received surgery, liver transplantation or sorafenib after TACE; 
(2) patients with synchronous malignant tumours; (3) patients who were lost to follow-up within six months; and (4) patients with incomplete baseline data such as incomplete liver function test results and incomplete TNM information. The validation cohort I included those patients with a confirmed diagnosis of HCC who received supportive care.

The patients received TACE treatments after multiple disciplinary team discussions. We regularly followed up patients and evaluated their treatment response three to four weeks after TACE with contrast-enhanced spiral computed tomography. If necessary, we repeated the TACE procedures.

We obtained written informed consent from patients or their family members before the study. The institutional ethics committee approved this study, and the study observed the 1964 Helsinki Declaration.

\section{Data collection}

We reviewed and retrieved the patients' clinical data from the hospital database. We collected medical history, blood routine examination, biochemical examination, tumour markers and $\mathrm{CT} / \mathrm{MR}$ results before the first TACE procedure was administered. OS time was defined as the date of the initial diagnosis to the date of death or the date of the last follow-up.

In the training cohort, we collected the clinical demographics, pretreatment laboratory test results (routine blood test, liver function tests including ALB and ALP, renal function test, AFP, etc.) and tumour-related characteristics of the HCC patients. Tumour-related characteristics such as tumour size, node metastasis, distant metastasis and portal vein tumour thrombus (PVTT) were acquired from CT/MR. We substituted the Child-Pugh grade for the AAPR in the CLIP score to establish the AAPR-CLIP and integrated the AAPR into the TNM staging system to establish the AAPR-TNM. Then, the clinical staging of each patient was correspondingly performed in accordance with the AAPR-CLIP, AAPR-TNM, CLIP and TNM staging systems. Next, the prognostic value of the AAPR was confirmed in both validation cohorts I and II.

\section{Statistical analysis}

We compared the difference of categorical variables between groups by Chi-square test and Fisher's exact test (two-tailed). We compared the differences between medians by the Mann-Whitney test. We compared the means and standard errors of continuous variables by Student's $t$ test. We dichotomized the patients into a high-AAPR group or a low-AAPR group based on the best cut-off value of the AAPR. We explored the correlations of the AAPR levels and other clinical variables by Chi-square test. We identified the significant prognostic factors of OS in univariate analyses by the Kaplan-Meier method. Those significant prognostic factors identified in univariate analyses were further analysed in the Cox proportional hazards regression model to identify independent prognostic factors. A staging system would have a better discriminatory ability in stratifying patients with different prognosis if it had a larger AUC. We compared the prognostic abilities of the different staging systems in OS prediction by the likelihood ratio test (LRT) and the comparisons of Akaike information criterion (AIC) values. All of the analyses of the data were performed by using Medcalc (version 15.8; MedCalc Software bvba, Acacialaan, Belgium), SPSS (version 24.0; IBM Corp., Armonk, NY, USA) and SAS (version 9.1.3; SAS Institute, Inc., Cary, NC, USA).

\section{Results}

\section{Patient demographics}

Baseline demographics of the training cohort and validation cohorts I and II are shown in Table 1. The training cohort recruited 372 HCC patients. Their median age was 52 years (IQR 44-61 years). There were far more male patients than female ones [348 vs $24(93.5 \%$ vs $6.5 \%)]$. Seventy-seven $(20.7 \%)$ patients were diagnosed with ascites. Their median levels of AFP, BUN, ALB, TBIL, ALT, AST and ALP were $277.39 \mathrm{ng} / \mathrm{dL}, 5.05 \mathrm{mmol} / \mathrm{L}, 38.70 \mathrm{~g} / \mathrm{L}, 16.65 \mu \mathrm{mol} / \mathrm{L}$, $46 \mathrm{U} / \mathrm{L}, 55 \mathrm{U} / \mathrm{L}$ and $107 \mathrm{U} / \mathrm{L}$, respectively. A total of $286(76.9 \%)$ patients were classified as Child-Pugh grade $\mathrm{A}$, and $86(23.1 \%)$ patients were classified as grade B, while $126(33.9 \%)$ and $155(41.7 \%)$ patients were classified as T3 and T4 (AJCC $7^{\text {th }}$ ), respectively. Seventy-one $(19.1 \%)$ patients were found to have lymph node metastasis, and $30(8.1 \%)$ had distant metastasis, while $160(43.0 \%)$ patients were diagnosed with PVTT. The 3-month, 6-month, 1-year and 2-year survival rates of these patients were $91.1 \%, 82.0 \%$, $68.5 \%$ and $54.6 \%$, respectively.

We compared the pretreatment levels of the AAPR between the subgroups. The level of the AAPR was significantly higher among patients without PVTT than those with PVTT $(0.405 \pm 0.013$ vs $0.321 \pm$ 0.012 , respectively, $P<0.001)$, with T1-2 than those with T3-4 (0.416 \pm 0.020 vs $0.353 \pm 0.010$, respectively, $P=0.003)$, with N0 than those with N1 $(0.381 \pm 0.010$ vs $0.318 \pm 0.017$, respectively, $P=0.006)$, and with TNM stage I-II than those with TNM stage III-IV $(0.414 \pm 0.020$ vs $0.355 \pm 0.010$, respectively, $P=0.005)$ (Figure 1). 
Table 1: Baseline demographics and clinical characteristics of the three independent cohorts in the current study.

\begin{tabular}{|c|c|c|c|c|}
\hline \multicolumn{2}{|l|}{ Characteristics } & $\begin{array}{l}\text { Training } \\
\text { cohort, } \mathbf{n}(\%)\end{array}$ & $\begin{array}{l}\text { Validation } \\
\text { cohort I, n (\%) }\end{array}$ & $\begin{array}{l}\text { Validation } \\
\text { cohort II, n (\%) }\end{array}$ \\
\hline \multicolumn{2}{|l|}{ Total } & $372(100.0)$ & 202(100.0) & $82(100.0)$ \\
\hline \multicolumn{2}{|c|}{ Age, years (median/IQR) } & $52(44-61)$ & $56(45-65)$ & $55(44-63)$ \\
\hline \multirow[t]{2}{*}{ Gender } & Male & $348(93.5)$ & $176(87.1)$ & $73(89.0)$ \\
\hline & Female & $24(6.5)$ & $26(12.9)$ & $9(11.0)$ \\
\hline \multirow[t]{2}{*}{ Ascites } & Yes & $77(20.7)$ & $113(55.9)$ & $17(20.7)$ \\
\hline & No & $295(79.3)$ & $89(44.1)$ & $65(79.3)$ \\
\hline \multicolumn{5}{|c|}{ Laboratory parameters (median/IQR) } \\
\hline \multicolumn{2}{|c|}{$\mathrm{AFP}, \mathrm{ng} / \mathrm{mL}$} & $\begin{array}{l}277.39 \\
(19.45-1000.00)\end{array}$ & $\begin{array}{l}1000 \\
(22.78-1210.00)\end{array}$ & $\begin{array}{l}34.69 \\
(8.28-1000.00)\end{array}$ \\
\hline \multicolumn{2}{|l|}{$\mathrm{BUN}, \mathrm{mmol} / \mathrm{L}$} & $5.05(4.06-5.95)$ & $5.11(3.99-7.14)$ & $4.47(3.87-5.64)$ \\
\hline \multicolumn{2}{|l|}{$\mathrm{ALB}, \mathrm{g} / \mathrm{L}$} & $\begin{array}{l}38.70 \\
(34.63-42.00)\end{array}$ & $\begin{array}{l}34.60 \\
(29.55-38.80)\end{array}$ & $\begin{array}{l}36.75 \\
(32.45-40.78)\end{array}$ \\
\hline \multicolumn{2}{|l|}{ TBIL, $\mu \mathrm{mol} / \mathrm{L}$} & $\begin{array}{l}16.65 \\
(12.20-25.23)\end{array}$ & $\begin{array}{l}31.05 \\
(17.18-71.88)\end{array}$ & $\begin{array}{l}15.95 \\
(11.93-24.28)\end{array}$ \\
\hline \multicolumn{2}{|l|}{$\mathrm{ALT}, \mathrm{U} / \mathrm{L}$} & $\begin{array}{l}46.00 \\
(33.00-70.75)\end{array}$ & NA & $44.5(31.75-68.75)$ \\
\hline \multicolumn{2}{|l|}{ AST, U/L } & $\begin{array}{l}55.00 \\
(38.25-95.25)\end{array}$ & $\begin{array}{l}134 \\
(72.00-228.00)\end{array}$ & $53.5(36.75-74.00)$ \\
\hline \multicolumn{2}{|l|}{$\mathrm{ALP}, \mathrm{U} / \mathrm{L}$} & $\begin{array}{l}107.00 \\
(82.00-155.75)\end{array}$ & $154(100-235.75)$ & $\begin{array}{l}108.5 \\
(84.5-153.75)\end{array}$ \\
\hline \multirow[t]{3}{*}{ Child-Pugh grade } & A & $286(76.9)$ & $54(26.7)$ & $79(96.3)$ \\
\hline & B & $86(23.1)$ & $100(49.5)$ & $3(3.7)$ \\
\hline & $\mathrm{C}$ & $0(0)$ & $48(23.8)$ & $0(0)$ \\
\hline \multirow{4}{*}{$\begin{array}{l}\text { NCCN-TNM stage } \\
\text { (AJCC 7th) }\end{array}$} & I & $21(5.6)$ & $20(9.9)$ & $7(8.5)$ \\
\hline & II & $67(18.0)$ & $13(6.4)$ & $20(24.4)$ \\
\hline & III & $213(57.3)$ & $120(59.4)$ & $29(35.4)$ \\
\hline & IV & $71(19.1)$ & $49(24.3)$ & $26(31.7)$ \\
\hline \multirow{4}{*}{$\begin{array}{l}\text { T category (AJCC } \\
7 \text { th) }\end{array}$} & $\mathrm{T} 1$ & $21(5.6)$ & $21(10.4)$ & $7(8.5)$ \\
\hline & $\mathrm{T} 2$ & $70(18.8)$ & $18(8.9)$ & $21(25.6)$ \\
\hline & $\mathrm{T} 3$ & $126(33.9)$ & $151(74.8)$ & $43(52.4)$ \\
\hline & $\mathrm{T} 4$ & $155(41.7)$ & $12(5.9)$ & $11(13.5)$ \\
\hline \multirow{2}{*}{$\begin{array}{l}\mathrm{N} \text { category (AJCC } \\
7 \text { th) }\end{array}$} & No & $301(80.9)$ & $143(70.8)$ & $61(74.4)$ \\
\hline & N1 & 71 (19.1) & $59(29.2)$ & $21(25.6)$ \\
\hline \multirow{2}{*}{$\begin{array}{l}\text { M category (AJCC } \\
7 \text { th) }\end{array}$} & M0 & $342(91.9)$ & $156(77.2)$ & 77 (93.9) \\
\hline & M1 & $30(8.1)$ & $46(22.8)$ & $5(6.1)$ \\
\hline PVTT & Yes & $160(43.0)$ & $143(70.8)$ & $66(80.5)$ \\
\hline & No & $212(57.0)$ & $59(29.2)$ & $16(19.5)$ \\
\hline AAPR-TNM & 1 & $10(2.7)$ & $4(2.0)$ & $2(2.5)$ \\
\hline & 2 & $38(10.2)$ & $17(8.4)$ & $11(13.4)$ \\
\hline & 3 & $107(28.8)$ & $22(10.9)$ & $22(26.8)$ \\
\hline & 4 & 159 (42.7) & $116(57.4)$ & $27(32.9)$ \\
\hline & 5 & $58(15.6)$ & $43(21.3)$ & $20(24.4)$ \\
\hline CLIP & 0 & $56(15.1)$ & $2(1.0)$ & $12(14.6)$ \\
\hline & 1 & 77 (20.7) & $15(7.4)$ & $16(19.5)$ \\
\hline & 2 & $83(22.3)$ & $26(12.9)$ & $26(31.7)$ \\
\hline & 3 & $68(18.3)$ & 39 (19.3) & $18(22.0)$ \\
\hline & 4 & $79(21.2)$ & $52(25.7)$ & $10(12.2)$ \\
\hline & 5 & $9(2.4)$ & $51(25.3)$ & $0(0)$ \\
\hline & 6 & $0(0)$ & $17(8.4)$ & $0(0)$ \\
\hline AAPR-CLIP & 0 & $32(8.6)$ & $1(0.5)$ & $5(6.1)$ \\
\hline & 1 & $81(21.8)$ & $8(4.0)$ & $11(13.4)$ \\
\hline & 2 & $61(16.4)$ & $10(5.0)$ & $18(22.0)$ \\
\hline & 3 & 71 (19.1) & $32(15.8)$ & $26(31.7)$ \\
\hline & 4 & 74 (19.9) & 33 (16.3) & $13(15.8)$ \\
\hline & 5 & 53 (14.2) & $51(25.2)$ & $9(11.0)$ \\
\hline & 6 & $0(0)$ & $50(24.8)$ & $0(0)$ \\
\hline & 7 & $0(0)$ & $17(8.4)$ & $0(0)$ \\
\hline Survival rates & 3-month & 339 (91.1) & 86 (42.6) & $81(98.8)$ \\
\hline & 6-month & $305(82.0)$ & 43 (21.3) & $71(86.6)$ \\
\hline & 1-year & $255(68.5)$ & $12(5.9)$ & $61(74.4)$ \\
\hline & 2-year & $203(54.6)$ & $3(1.5)$ & $49(59.8)$ \\
\hline & 5-year & $95(25.5)$ & $0(0)$ & $19(23.2)$ \\
\hline
\end{tabular}

HCC: hepatocellular carcinoma; TACE: transcatheter arterial chemoembolization; IQR: interquartile range; AFP: alpha fetoprotein; BUN: blood urea nitrogen; ALB: albumin; TBIL: total bilirubin; ALT: alanine aminotransferase; AST: aspartate aminotransferase; ALP: alkaline phosphatase; AAPR: albumin-to-alkaline phosphatase ratio; PVTT: portal vein tumour thrombus; NCCN: The National Comprehensive Cancer Network; AJCC: The American Joint Committee on Cancer; TNM: tumour-node-metastasis; CLIP: Cancer of the Liver Italian Program.

\section{Comparisons of HCC patients with high- and low-AAPR in the training cohort}

We confirmed the best cut-off value for AAPR was 0.439 with a sensitivity of $74.6 \%$ and a specificity of $40.7 \%$ by ROC curve analysis. Based on the cut-off value, the patients were classified into either the high-AAPR group or the low-AAPR group; thus, 117 $(31.5 \%)$ and $255(68.5 \%)$ patients were included in the high-AAPR group and the low-AAPR group, respectively.

The correlations between the AAPR and other clinicopathologic variables were explored by Chi-square analysis. As shown in Table 2, compared to patients in the low-AAPR group, the patients in the high-AAPR group had significantly higher frequencies of Child-Pugh grade A, TNM stage I-II, $\mathrm{T} 1-\mathrm{T} 2, \mathrm{ALP} \leq 200 \mathrm{U} / \mathrm{L}$ and ALB $>36 \mathrm{~g} / \mathrm{L}$, but lower frequencies of node metastasis and PVTT $(P<0.05)$.

Table 2: The Chi-square analysis of the clinicopathologic variables between the high-AAPR and low-AAPR groups in the training cohort.

\begin{tabular}{|c|c|c|c|c|}
\hline \multicolumn{2}{|l|}{ Variables } & \multirow{2}{*}{$\begin{array}{l}\begin{array}{l}\text { AAPR }>0.439, n \\
(\%)\end{array} \\
117(31.5)\end{array}$} & \multirow{2}{*}{$\begin{array}{l}\text { AAPR } \leq 0.439, \mathrm{n} \\
(\%) \\
255(68.5)\end{array}$} & \multirow[t]{2}{*}{$P$ value } \\
\hline Total & & & & \\
\hline \multirow[t]{2}{*}{ Age, years } & $\leq 52$ & $51(43.6)$ & $135(52.9)$ & 0.094 \\
\hline & $>52$ & $66(56.4)$ & $120(47.1)$ & \\
\hline \multirow[t]{2}{*}{ Gender } & Male & $110(94.0)$ & $238(93.3)$ & 0.803 \\
\hline & Female & $7(6.0)$ & $17(6.7)$ & \\
\hline \multirow[t]{2}{*}{ Ascites } & Yes & $18(15.4)$ & $59(23.1)$ & 0.087 \\
\hline & No & 99 (84.6) & $196(76.9)$ & \\
\hline \multicolumn{5}{|c|}{ Laboratory parameters } \\
\hline \multirow[t]{2}{*}{$\mathrm{AFP}, \mathrm{ng} / \mathrm{dL}$} & $>400$ & $47(40.2)$ & $126(49.4)$ & 0.097 \\
\hline & $\leq 400$ & $70(59.8)$ & $129(50.6)$ & \\
\hline \multirow[t]{2}{*}{$\mathrm{BUN}, \mathrm{mmol} / \mathrm{L}$} & $>8.9$ & $3(2.6)$ & $8(3.1)$ & 0.762 \\
\hline & $\leq 8.9$ & $114(97.4)$ & $247(96.9)$ & \\
\hline \multirow[t]{2}{*}{ ALB, g/L } & $>36$ & $107(91.5)$ & $139(54.5)$ & $<0.001$ \\
\hline & $\leq 36$ & $10(8.5)$ & $116(45.5)$ & \\
\hline \multirow[t]{2}{*}{$\mathrm{ALP}, \mathrm{U} / \mathrm{L}$} & $>200$ & $0(0)$ & $51(20.0)$ & $<0.001$ \\
\hline & $\leq 200$ & $117(100.0)$ & $204(80.0)$ & \\
\hline \multirow[t]{2}{*}{ Child-Pugh grade } & A & $104(88.9)$ & $182(71.4)$ & $<0.001$ \\
\hline & B & $13(11.1)$ & $73(28.6)$ & \\
\hline \multirow[t]{2}{*}{ TNM stage } & III-IV & $80(68.4)$ & $204(80.0)$ & 0.014 \\
\hline & I-II & 37 (31.6) & $51(20.0)$ & \\
\hline \multirow[t]{2}{*}{$\mathrm{T}$ category } & T3-4 & $79(67.5)$ & $202(79.2)$ & 0.015 \\
\hline & $\mathrm{T} 1-2$ & $38(32.5)$ & $53(20.8)$ & \\
\hline \multirow[t]{2}{*}{ N category } & No & $105(89.7)$ & $196(76.9)$ & 0.003 \\
\hline & N1 & $12(10.3)$ & $59(23.1)$ & \\
\hline \multirow[t]{2}{*}{ M category } & M0 & $112(95.7)$ & $230(90.2)$ & 0.069 \\
\hline & M1 & $5(4.3)$ & $25(9.8)$ & \\
\hline \multirow[t]{2}{*}{ PVTT } & Yes & $29(24.8)$ & $131(51.4)$ & $<0.001$ \\
\hline & No & 88 (75.2) & $124(48.6)$ & \\
\hline \multirow[t]{4}{*}{ Survival rates $(\%)$} & 3-months & 99.1 & 87.5 & $<0.001$ \\
\hline & 6-months & 94.0 & 76.5 & $<0.001$ \\
\hline & 1-year & 85.5 & 60.8 & $<0.001$ \\
\hline & 2-year & 71.8 & 46.7 & $<0.001$ \\
\hline
\end{tabular}

HCC: hepatocellular carcinoma; TACE: transcatheter arterial chemoembolization; AFP: alpha fetoprotein; BUN: blood urea nitrogen; ALB: albumin; ALP: alkaline phosphatase; AAPR: albumin-to-alkaline phosphatase ratio; TNM:

tumour-node-metastasis; PVTT: portal vein tumour thrombus.

We compared the clinical parameters of 372 HCC patients in the training cohort. The patients in the high-AAPR group had significantly lower levels 

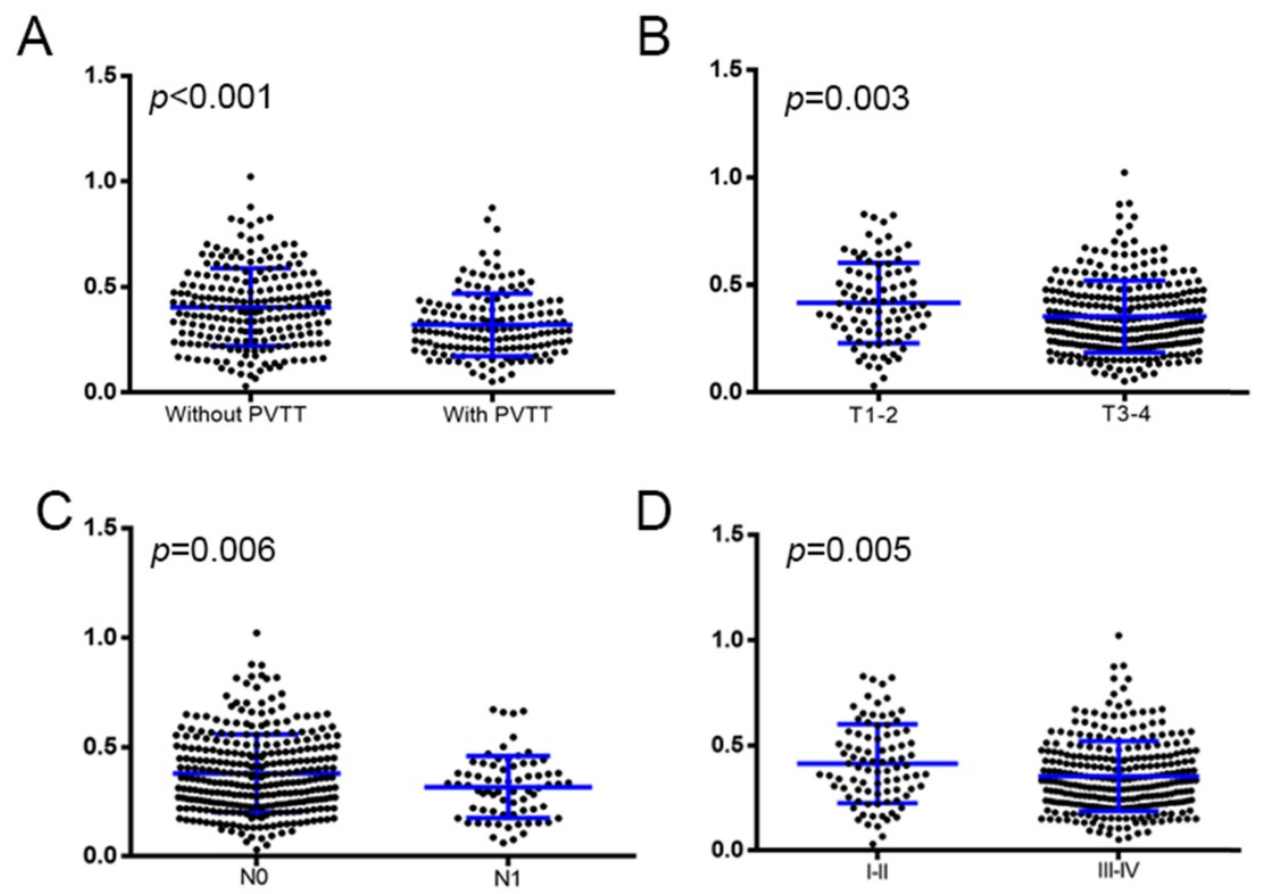

Figure 1. The pretreatment levels of the AAPR were compared between subgroups dichotomized by: (A) PVTT (without vs with); (B) T1-2 vs. T3-4; (C) N0 vs. N1; and (D) TNM stage I-II vs. III-IV. PVTT: portal vein tumour thrombus. N: node status. M: metastasis status. TNM: tumour-node-metastasis.

of alanine aminotransferase (ALT), aspartate aminotransferase (AST), alkaline phosphatase (ALP) and total bilirubin (TBIL) compared to the low-AAPR group $(42.77 \pm 2.485$ vs. $67.99 \pm 3.445, P<0.001 ; 50.99 \pm$ 3.521 vs. $93.60 \pm 5.924, P<0.001 ; 73.22 \pm 1.270$ vs. 161.0 $\pm 6.190, P<0.001 ; 17.23 \pm 0.709$ vs. $22.19 \pm 1.053, P=$ 0.0025 , respectively). The patients in the high-AAPR group showed a significantly higher level of albumin (ALB) than patients in the low-AAPR group (40.91 \pm 0.3710 vs. $36.84 \pm 0.3140$, respectively, $P<0.001)$. The levels of blood urea nitrogen (BUN) and alpha fetoprotein (AFP) were similar between the high- and low-AAPR groups (5.185 \pm 0.1495 vs. $5.193 \pm 0.1058, P$ $=0.965 ; 554.4 \pm 141.1$ vs. $563.5 \pm 32.35, P=0.9319$, respectively).

\section{Univariate and multivariate analyses of prognostic factors for OS}

In the training cohort, the 3-month, 6-month, 1-year and 2-year survival rates were 99.1\%, 94.0\%, $85.5 \%$ and $71.8 \%$ for patients in the high-AAPR group, while they were $87.5 \%, 76.5 \%, 60.8 \%$ and $46.7 \%$ for those in the low-AAPR group, respectively. The median OS for the patients in the high-AAPR group was significantly longer than for those in the low-AAPR group (58.4 m vs. $17.8 \mathrm{~m}$, respectively, $P<$ 0.001). We carried out both univariate and multivariate analyses of the potential prognostic factors to identify whether the AAPR was an independent prognostic factor for OS. In univariate analysis, clinical variables including age, gender, ascites, AFP, BUN, ALB, ALP, AAPR, T category, N category, M category, TNM stage and PVTT were evaluated. As shown in Figure 2, the OS was significantly different between the subgroups classified by the AFP, AAPR, TNM, PVTT, T category and $M$ category $(P<0.05)$. Subsequently, we used the above variates in the Cox proportional hazards model to further distinguish among the independent prognostic factors for OS in multivariate analysis. Then, the AAPR was identified as an independent prognostic factor for $\mathrm{OS}(H R=0.636, P=0.003)$ together with the PVTT and the distant metastasis $(H R=1.781, P=0.003$ for PVTT $H R=1.916, P=0.004$ for $\mathrm{M}$ category) (Table 3$)$.

The pretreatment levels of ALB, ALP and the Child-Pugh grade have been previously reported as prognostic factors for HCC patients. We compared the prognostic abilities of the ALB, ALP, Child-Pugh grade and AAPR in predicting the OS by ROC analysis. As shown in Figure 3, the AUC of the AAPR was larger than that of the ALB, ALP or Child-Pugh grade $(0.574,0.525,0.513$ and 0.525 , respectively), suggesting that the AAPR had better prognostic ability compared with the ALB, ALP and Child-Pugh grades for predicting OS among the $372 \mathrm{HCC}$ patients treated with TACE.

We further confirmed the prognostic value of the AAPR in validation cohorts I and II. Both univariate and multivariate analyses identified the AAPR as an independent prognostic factor for OS in these two cohorts $(H R=0.468, P=0.014 ; H R=0.349, P<0.001)$ (Tables 4 and 5). 


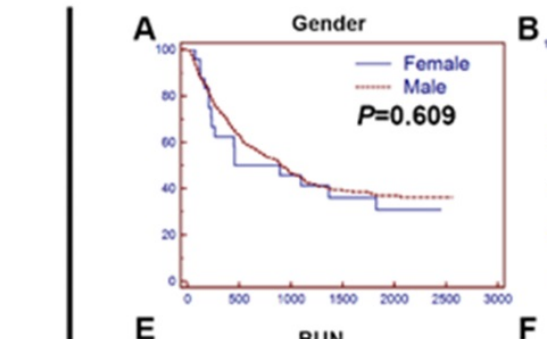

E
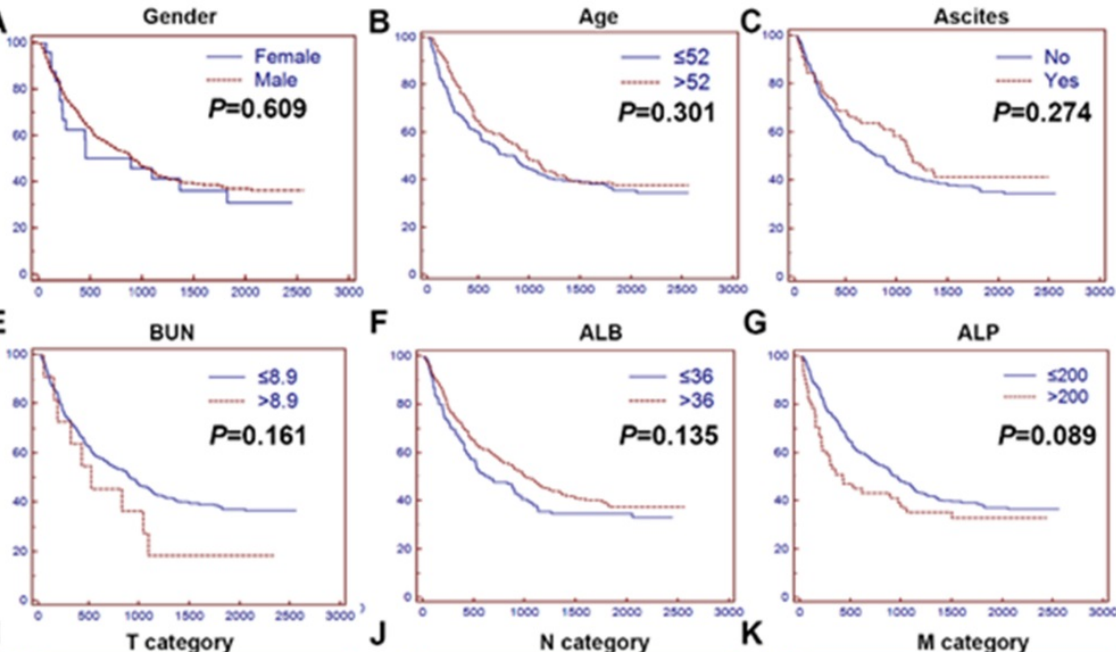

G
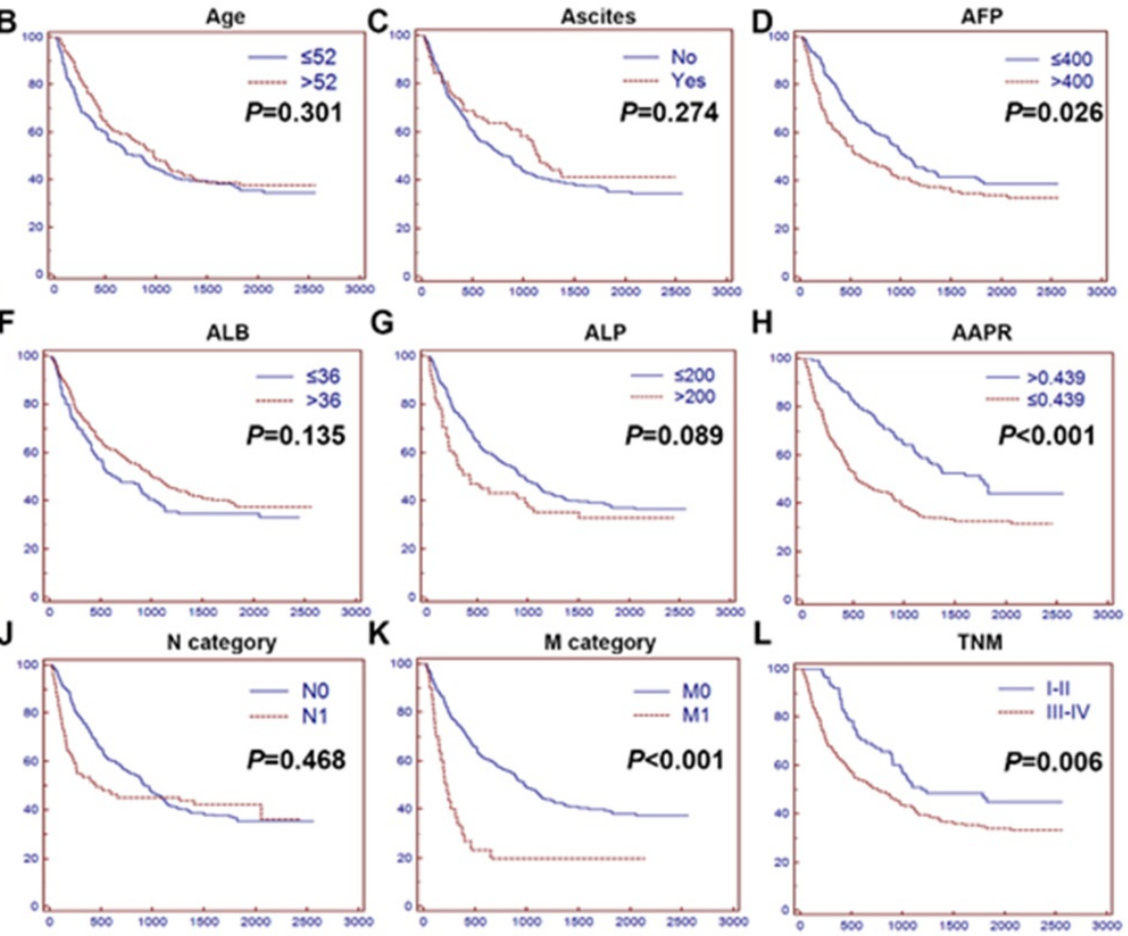

H
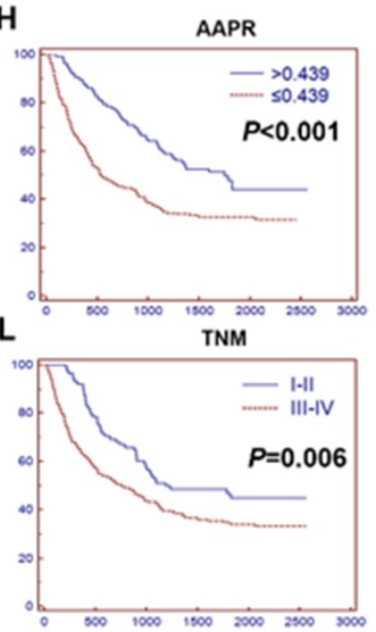

M

PVTT

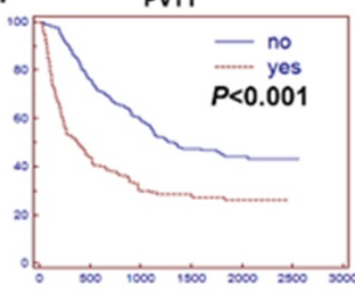

\section{Time after diagnosis(days)}

Figure 2. The Kaplan-Meier curves for overall survival (OS) were stratified by different features of 372 HCC patients treated with TACE. The curves above were stratified by gender (A), age (B), ascites (C), AFP, ng/mL (D), BUN, mmol/L (E), ALB, g/L (F), ALP, U/L (G), AAPR (H), T category (AJCC 7th) (I), N category (AJCC 7th) (J), M category (AJCC 7th) (K), TNM (L) and PVTT (M). The OS was significantly different between subgroups stratified by AFP, AAPR, TNM, PVTT, T category and M category ( $<$ < 0.05$)$. AFP: alpha fetoprotein; BUN: blood urea nitrogen; ALB: albumin; ALP: alkaline phosphatase; AJCC: American Joint Committee on Cancer; N: node status. M: metastasis status. TNM: tumour-node-metastasis. AAPR: albumin-to-alkaline phosphatase ratio; PVTT: portal vein tumour thrombus

Table 3: Univariate and multivariate analyses of the prognostic factors for overall survival in 372 HCC patients treated with TACE.

\begin{tabular}{|c|c|c|c|c|c|c|c|}
\hline \multirow[t]{2}{*}{ Variables } & \multicolumn{2}{|l|}{ Univariate } & \multicolumn{5}{|c|}{ Multivariate } \\
\hline & Log-rank $\chi 2$ & $P$ value & $\mathrm{B}$ & SE & HR & $95 \% \mathrm{CI}$ & $P$ value \\
\hline Age, years $(>52 / \leq 52)$ & 1.068 & 0.301 & & & & & \\
\hline Gender (male/female) & 0.262 & 0.609 & & & & & \\
\hline Ascites (yes/no) & 1.196 & 0.274 & & & & & \\
\hline \multicolumn{8}{|l|}{ Laboratory parameters } \\
\hline ALP, U/L $(>200 / \leq 200)$ & 2.898 & 0.089 & & & & & \\
\hline ALB, $g / L(>36 / \leq 36)$ & 2.234 & 0.135 & & & & & \\
\hline $\mathrm{AFP}, \mathrm{ng} / \mathrm{dL}(>400 / \leq 400)$ & 4.947 & $0.026^{*}$ & 0.132 & 0.138 & 1.141 & 0.873 to 1.493 & 0.337 \\
\hline $\mathrm{BUN}, \mathrm{mmol} / \mathrm{L}(>8.9 / \leq 8.9)$ & 1.967 & 0.161 & & & & & \\
\hline $\mathrm{T}$ category $(\mathrm{T} 1-2 / \mathrm{T} 3-4)$ & 7.412 & $0.006^{*}$ & -0.004 & 0.733 & 0.996 & 0.238 to 4.160 & 0.996 \\
\hline $\mathrm{N}$ category (N0/N1) & 0.527 & 0.468 & & & & & \\
\hline M category (M0/M1) & 18.400 & $<0.001^{*}$ & 0.650 & 0.228 & 1.916 & 1.229 to 2.987 & 0.004 \\
\hline TNM (I-II/III-IV) & 7.670 & $0.006^{*}$ & 0.042 & 0.744 & 1.042 & 0.245 to 4.443 & 0.955 \\
\hline PVTT (yes/no) & 33.300 & $<0.001^{*}$ & 0.577 & 0.155 & 1.781 & 1.318 to 2.408 & $<0.001$ \\
\hline Child-Pugh grade (A/B) & 0.612 & 0.434 & & & & & \\
\hline AAPR $(>0.439 / \leq 0.439)$ & 17.336 & $<0.001^{*}$ & -0.453 & 0.154 & 0.636 & 0.471 to 0.858 & 0.003 \\
\hline
\end{tabular}

HCC: hepatocellular carcinoma; TACE: transcatheter arterial chemoembolization; ALP: alkaline phosphatase; ALB: albumin; AFP: alpha fetoprotein; BUN: blood urea nitrogen; TNM: tumour-node-metastasis; PVTT: portal vein tumour thrombus; AAPR: albumin-to-alkaline phosphatase ratio.

* Variables with a $P$ value less than 0.05 were entered into multivariate analysis. 


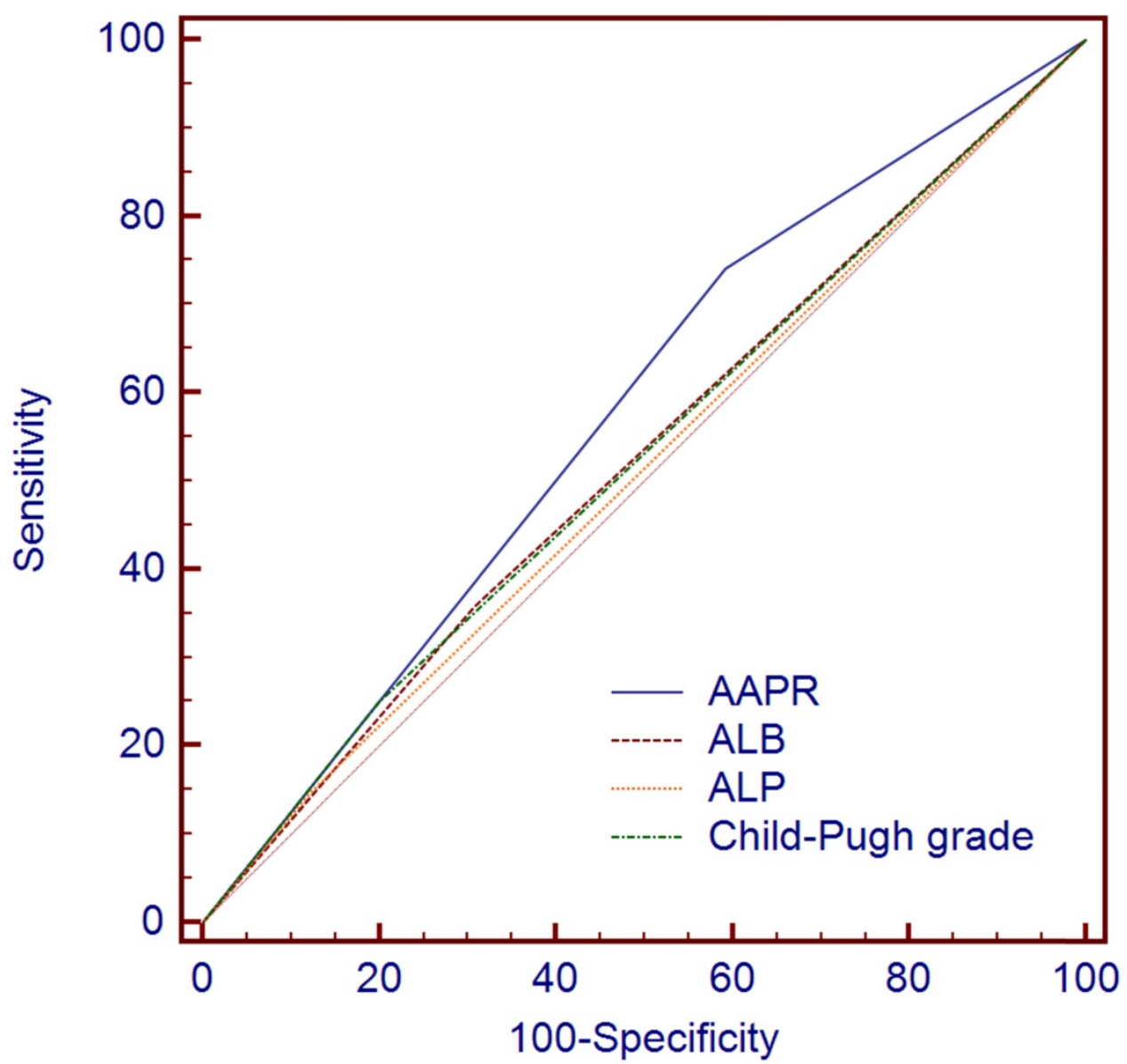

Figure 3. The comparisons of the area under curves (AUCs) for survival status among AAPR, ALB and ALP using receiver operating characteristic curves (ROC curves). The AUCs of AAPR, ALB, ALP and Child-Pugh grade were $0.574,0.525,0.513$ and 0.525 , respectively. AAPR: albumin-to-alkaline phosphatase ratio; ALB: albumin; ALP: alkaline phosphatase.

Table 4: Univariate and multivariate analyses of prognostic factors for overall survival in validation cohort I.

\begin{tabular}{|c|c|c|c|c|c|c|c|}
\hline \multirow[t]{2}{*}{ Variables } & \multicolumn{2}{|l|}{ Univariate } & \multicolumn{5}{|c|}{ Multivariate } \\
\hline & Log-rank $\chi 2$ & $P$ value & B & SE & HR & $95 \% \mathrm{CI}$ & $P$ value \\
\hline Age, years $(>56 / \leq 56)$ & 0.442 & 0.506 & & & & & \\
\hline Gender (male/female) & 0.016 & 0.899 & & & & & \\
\hline Ascites (yes/no) & 11.315 & $0.001^{*}$ & -0.156 & 0.195 & 0.856 & 0.586 to 1.250 & 0.423 \\
\hline \multicolumn{8}{|l|}{ Laboratory parameters } \\
\hline ALP, U/L (> 200/ $\leq 200)$ & 6.926 & $0.008^{*}$ & 0.002 & 0.177 & 1.002 & 0.709 to 1.416 & 0.991 \\
\hline $\mathrm{ALB}, \mathrm{g} / \mathrm{L}(>36 / \leq 36)$ & 1.692 & 0.193 & & & & & \\
\hline $\mathrm{AFP}, \mathrm{ng} / \mathrm{dL}(>400 / \leq 400)$ & 9.048 & $0.003^{*}$ & 0.268 & 0.164 & 1.307 & 0.950 to 1.799 & 0.102 \\
\hline $\mathrm{BUN}, \mathrm{mmol} / \mathrm{L}(>8.9 / \leq 8.9)$ & 6.608 & $0.010^{*}$ & 0.725 & 0.226 & 2.064 & 1.329 to 3.206 & 0.001 \\
\hline T category (T1-2/T3-4) & 11.450 & $0.001^{*}$ & -0.252 & 0.476 & 0.778 & 0.307 to 1.969 & 0.597 \\
\hline $\mathrm{N}$ category $(\mathrm{N} 0 / \mathrm{N} 1)$ & 3.384 & 0.066 & & & & & \\
\hline M category (M0/M1) & 0.093 & 0.761 & & & & & \\
\hline TNM (I-II/III-IV) & 11.280 & $0.001^{*}$ & 0.895 & 0.538 & 2.447 & 0.858 to 6.980 & 0.096 \\
\hline PVTT (yes/no) & 25.406 & $<0.001^{*}$ & 0.502 & 0.224 & 1.652 & 1.069 to 2.555 & 0.025 \\
\hline Child-Pugh grade & 23.713 & $<0.001^{*}$ & & & & & \\
\hline A & & & NA & NA & 1 & Reference & NA \\
\hline B & & & 0.385 & 0.217 & 1.470 & 0.962 to 2.246 & 0.076 \\
\hline $\mathrm{C}$ & & & 1.061 & 0.290 & 2.889 & 1.640 to 5.089 & $<0.001$ \\
\hline AAPR $(>0.439 / \leq 0.439)$ & 19.729 & $<0.001^{*}$ & -0.759 & 0.309 & 0.468 & 0.256 to 0.855 & 0.014 \\
\hline
\end{tabular}

HCC: hepatocellular carcinoma; TACE: transcatheter arterial chemoembolization; ALP: alkaline phosphatase; ALB: albumin; AFP: alpha fetoprotein; BUN: blood urea nitrogen; TNM: tumour-node-metastasis; PVTT: portal vein tumour thrombus; AAPR: albumin-to-alkaline phosphatase ratio; NA: not applicable.

* Variables with a $P$ value less than 0.05 were entered into multivariate analysis. 
Table 5. Univariate and multivariate analyses of prognostic factors for overall survival in validation cohort II.

\begin{tabular}{|c|c|c|c|c|c|c|c|}
\hline \multirow[t]{2}{*}{ Variables } & \multicolumn{2}{|l|}{ Univariate } & \multicolumn{5}{|c|}{ Multivariate } \\
\hline & Log-rank $\chi 2$ & $P$ value & $\mathrm{B}$ & SE & HR & $95 \% \mathrm{CI}$ & $P$ value \\
\hline Age, years $(>55 / \leq 55)$ & 0.665 & 0.415 & & & & & \\
\hline Gender (male/female) & 0.525 & 0.469 & & & & & \\
\hline Ascites (yes/no) & 0.026 & 0.872 & & & & & \\
\hline \multicolumn{8}{|l|}{ Laboratory parameters } \\
\hline ALP, U/L $(>200 / \leq 200)$ & 7.166 & $0.007^{*}$ & 0.333 & 0.656 & 1.395 & 0.388 to 5.009 & 0.612 \\
\hline $\mathrm{ALB}, \mathrm{g} / \mathrm{L}(>36 / \leq 36)$ & 3.871 & $0.049^{*}$ & -0.455 & 0.526 & 0.634 & 0.228 to 1.767 & 0.386 \\
\hline $\mathrm{AFP}, \mathrm{ng} / \mathrm{dL}(>400 / \leq 400)$ & 0.089 & 0.765 & & & & & \\
\hline $\mathrm{BUN}, \mathrm{mmol} / \mathrm{L}(>8.9 / \leq 8.9)$ & 43.877 & $<0.001^{*}$ & 1.526 & 2.789 & 1.542 & 0.153 to 5.676 & 0.956 \\
\hline $\mathrm{T}$ category $(\mathrm{T} 1-2 / \mathrm{T} 3-4)$ & 8.633 & $0.003^{*}$ & 0.538 & 2.561 & 1.713 & 0.257 to 6.556 & 0.999 \\
\hline $\mathrm{N}$ category (N0/N1) & 1.699 & 0.192 & & & & & \\
\hline M category (M0/M1) & 0.483 & 0.487 & & & & & \\
\hline TNM (I-II/III-IV) & 8.578 & $0.003^{*}$ & 2.248 & 2.562 & 1.467 & 0.434 to 5.235 & 0.999 \\
\hline PVTT (yes/no) & 2.587 & 0.108 & & & & & \\
\hline Child-Pugh grade (A/B) & 0.043 & 0.837 & & & & & \\
\hline AAPR $(>0.439 / \leq 0.439)$ & 17.336 & $<0.001^{*}$ & -0.517 & 0.254 & 0.349 & 0.159 to 0.798 & $<0.001$ \\
\hline
\end{tabular}

HCC: hepatocellular carcinoma; TACE: transcatheter arterial chemoembolization; ALP: alkaline phosphatase; ALB: albumin; AFP: alpha fetoprotein; BUN: blood urea nitrogen; TNM: tumour-node-metastasis; PVTT: portal vein tumour thrombus; AAPR: albumin-to-alkaline phosphatase ratio; NA: not applicable.

*Variables with a $P$ value less than 0.05 were entered into multivariate analysis.

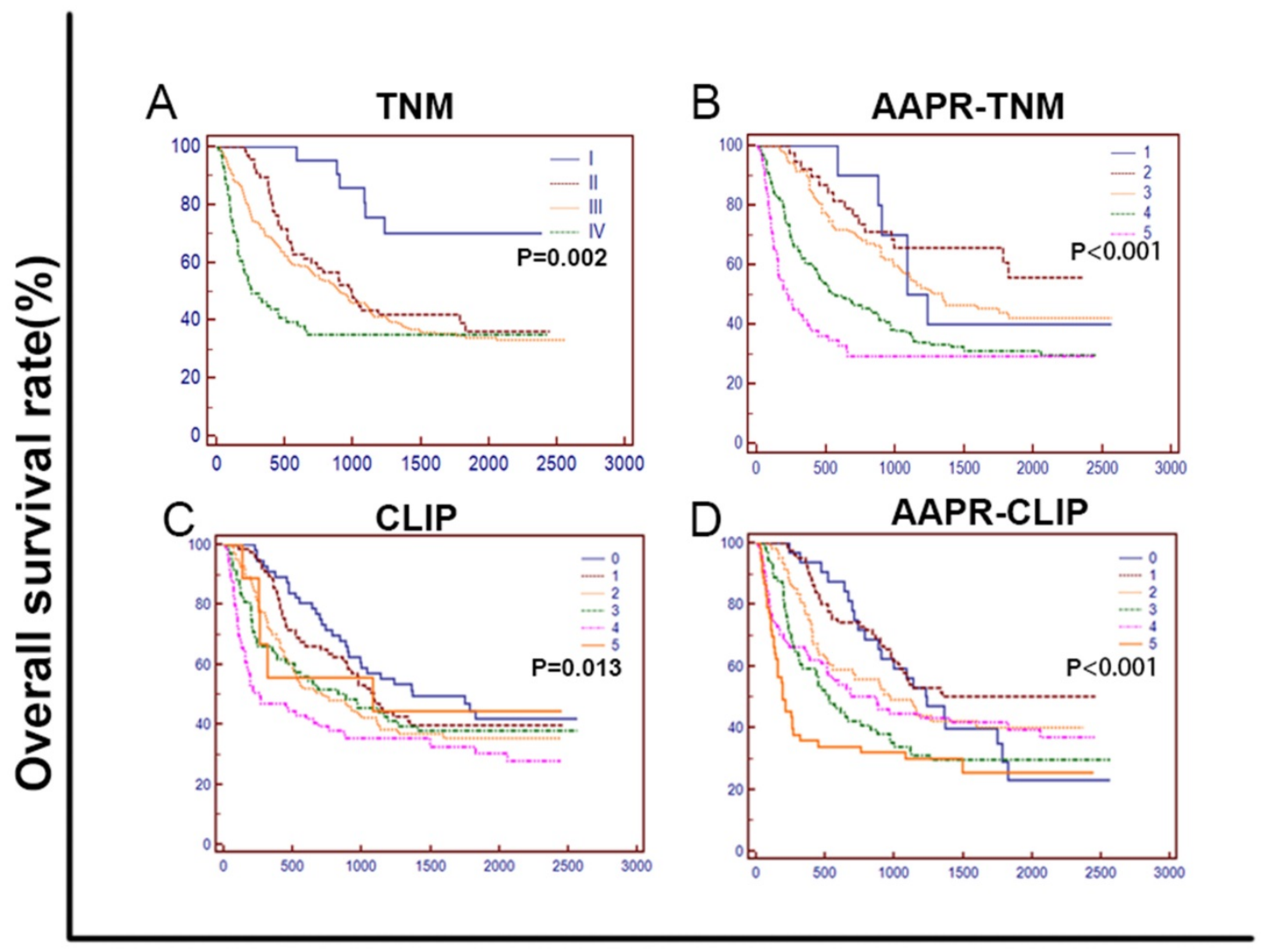

\section{Time after diagnosis(days)}

Figure 4. The Kaplan-Meier curves for OS of 372 HCC patients treated with TACE, which were stratified by TNM (A), AAPR-TNM (B), CLIP (C) and AAPR-CLIP (D). The OS was significantly different among subgroups stratified by the above variables $(P<0.05)$. TNM: tumour-node-metastasis; AAPR: albumin-to-alkaline phosphatase ratio; CLIP: Cancer of the Liver Italian Program.

\section{Comparison of the AAPR-CLIP, CLIP, AAPR-TNM and TNM staging systems}

To improve the prognostic ability of the CLIP, we substituted the AAPR for the Child-Pugh grade to establish the AAPR-CLIP (Supplemental Table 1). To improve the prognostic ability of the TNM, we integrated the AAPR into the TNM to establish the
AAPR-TNM. In the training cohort, as shown in Figure 4, the OS curves were stratified by the TNM, AAPR-TNM, CLIP and AAPR-CLIP $(P<0.05)$. When predicting 3-month, 6-month, 1-year and 2-year survival, the AUC of the AAPR-CLIP was significantly larger than that of both CLIP and TNM $(P<0.05)$. The AUC of the AAPR-TNM was 
significantly larger than that of the TNM $(P<0.05)$ (Figure 5 and Supplemental Table 2). When predicting OS, the LRT suggested that the AAPR-TNM had the largest $\chi^{2}$ and the smallest Akaike information criterion (AIC) values $(P<0.05)$. Specifically, the AAPR-CLIP had a significantly larger $X^{2}$ and a smaller AIC value than those of both TNM and CLIP $(P<0.05)$ (Table 6). The AAPR-TNM system was the

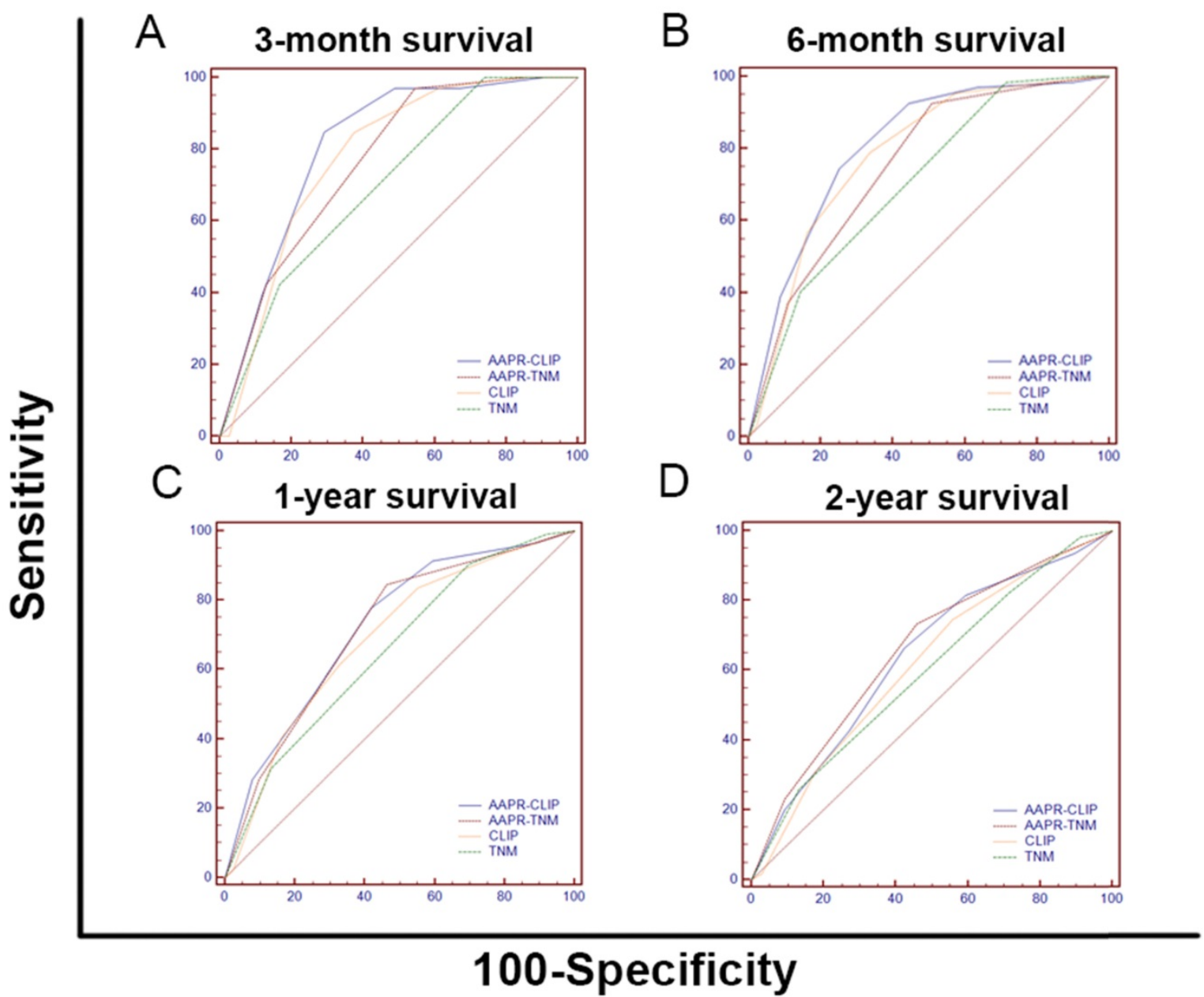

Figure 5. The receiver operating characteristic curves of the TNM, AAPR-TNM, CLIP and AAPR-CLIP for predicting 3-month, 6-month, 1-year and 2-year survival. (A) The AUCs of the AAPR-CLIP, AAPR-TNM, CLIP and TNM were $0.812,0.767,0.779$ and 0.703 , respectively, for predicting 3-month survival. (B) The AUCs of the AAPR-CLIP, AAPR-TNM, CLIP and TNM were 0.809, 0.756, 0.784 and 0.709, respectively, for predicting 6-month survival. (C) The AUCs of the AAPR-CLIP, AAPR-TNM, CLIP and TNM were $0.724,0.715,0.691$ and 0.656 , respectively, for predicting 1 -year survival. (D) The AUCs of the AAPR-CLIP, AAPR-TNM, CLIP and TNM were 0.639, 0.658, 0.614 and 0.593, respectively, for predicting 2-year survival. TNM: tumour-node-metastasis; AAPR: albumin-to-alkaline phosphatase ratio; CLIP: Cancer of the Liver Italian Program; AUC: area under the curve.

Table 6: The comparisons of the AAPR-CLIP, CLIP, AAPR-TNM and TNM staging systems for their values in prediction of overall survival in the training cohort and validation cohorts I and II

\begin{tabular}{lllllllll}
\hline Staging system & \multicolumn{2}{l}{ Training cohort } & \multicolumn{3}{l}{ Validation cohort I } & \multicolumn{2}{c}{ Validation cohort II } \\
\cline { 2 - 9 } & LRT $\chi^{2}$ & AIC & $P$ value & LRT $\chi^{2}$ & AIC & $P$ value & LRT $\chi^{2}$ & AIC \\
\hline AAPR-TNM & 27.15 & 2520.22 & $<0.001$ & 20.70 & 1617.77 & $<0.001$ & 23.52 & 141.78 \\
AAPR-CLIP & 16.95 & 2532.13 & $<0.001$ & 57.45 & 1578.11 & $<0.001$ & 10.11 & 161.88 \\
TNM & 14.39 & 2533.76 & $<0.001$ & 10.49 & 1629.55 & 0.001 & 5.84 & 166.60 \\
CLIP & 10.50 & 2538.54 & 0.001 & 54.91 & 1582.44 & $<0.001$ & 4.36 & 179.55
\end{tabular}

HCC: hepatocellular carcinoma; TACE: transcatheter arterial chemoembolization; TNM: tumour-node-metastasis; CLIP: The Cancer of the Liver Italian Program; LRT: likelihood ratio test; AIC: Akaike information criterion. AAPR: albumin-to-alkaline phosphatase ratio; NA: not applicable. best staging system in predicting OS among these four ing systems, followed by the AAPR-CLIP in the Both AAPR-TNM and AAPR-CLIP outperformed their corresponding primary system in predicting OS in the training cohort, validation cohorts I and II (Table 6). 


\section{Discussion}

Previously, our team confirmed AAPR as an independent prognostic index for OS among advanced HCC patients who were not receiving any standard anticancer therapy. However, the prognostic value of the AAPR for HCC patients treated with TACE remains unknown. Our current study identified the AAPR as an independent prognostic factor for predicting OS in a cohort of 372 patients who were diagnosed with HCC and treated with TACE, which was further confirmed in the validation cohort I and II. The AAPR-CLIP system preceded both the CLIP and TNM systems in predicting 3-month, 6-month, 1-year and 2-year survival in the training cohort. Both AAPR-TNM and AAPR-CLIP had better prognostic abilities than the TNM and CLIP in predicting OS in the training cohort, as shown in this study.

Many researchers have set out to identify potential independent prognostic factors in the routine blood tests and biochemical tests of cancer patients for their economical and convenient features. Several serum biomarkers such as the neutrophil to lymphocyte ratio (NLR), lactic dehydrogenase (LDH), ALB, ALP and AAPR have been reported as independent prognostic factors of OS in malignant tumours $[19,20]$.

The serum levels of ALB and ALP are two commonly used indicators of liver function in daily clinical practice. The serum ALB level is an important component of the Child-Pugh grade, which is commonly applied in evaluating liver function and has been integrated into many staging systems, such as the CLIP, BCLC and Japan Integrated Staging system (JIS). The serum ALB level can reflect the protein synthetic function of the liver and can also serve as a nutritional index. Hypoalbuminemia may reflect liver dysfunction and malnutrition, which eventually impairs the patients' immunity and results in a poor prognosis [21]. Albumin has been shown to inhibit the proliferation of hepatocellular carcinoma cells and to inhibit the phosphorylation of $\mathrm{Rb}$ proteins while increasing the expression of p21 and p57, leading to an increase in the G0/G1 cell population, which suppresses cell proliferation [22]. The ALP is a hydrolase enzyme that is mainly present in the liver, kidney and bone, etc. The serum ALP level was also identified as an independent prognostic factor of several malignant tumours, especially in patients with bone and liver metastasis. Certain subtypes of ALP have been found to be expressed in several tumour cell lines [23-25]. Therefore, an increased ALP level may reflect a heavy tumour burden or occurrence of distant metastasis, resulting in a poor prognosis. However, the molecular mechanisms of how ALP influences the prognosis of cancer patients needs to be further elucidated.

The AAPR has been confirmed as an independent prognostic factor of OS in HCC patients who received surgery or palliative therapies without any standard therapies, but not in patients treated with TACE. In our current study, a high AAPR level was associated with lower frequencies of PVTT, Child-Pugh grade B, T3-4, node metastasis and TNM III-IV stage. The median OS time of the patients in the high AAPR group was significantly longer than in the low-AAPR group. The patients in the high AAPR group had better prognosis. We also confirmed that the AAPR $\leq 0.439$ was associated with a poorer prognosis by both univariate and multivariate analyses. A low level of the AAPR may reflect liver dysfunction, malnutrition, a heavy tumour burden or tumour metastasis.

The AJCC TNM staging system has been widely used for HCC patients who received surgery and liver transplantation. However, the TNM has limited prognostic value for HCC patients treated with TACE and those with poor liver function because it only takes the tumour situation into account [26, 27]. Integrating the AAPR with the TNM and the CLIP may help to improve their prognostic abilities. In the current study, we found that both the AAPR-TNM and the AAPR-CLIP system were better than the TNM system in predicting 3-month, 6-month, 1-year, and 2-year survival OS in the training cohort, which provided a better stratification among HCC patients and might help when choosing treatment options. The median OS of the HCC patients with an AAPR-CLIP score of 5 was only 6.4 months, which means that they may need sorafenib, lenvatinib, cabozantinib or immune check point inhibitors in addition to TACE in order to improve their prognosis.

To the best of our knowledge, for the first time, we have identified AAPR as an independent prognostic factor for 372 HCC patients treated with TACE and confirmed the independent prognostic value of the AAPR in validation cohorts I and II. All of the patients in the training cohort and validation cohort II were treated with TACE, so we excluded the potential effects of other treatment options on their prognosis. Integrating AAPR into a widely used staging system such as TNM and CLIP may improve the prognostic abilities of both TNM and CLIP. Last but not least, the AAPR is an objective variable with discriminatory ability and is easy to calculate and apply in clinical practice.

However, our study has some limitations. First, our study was a retrospective study, and all of the patients were from one hospital and were all ethnically Chinese patients. Thus, these results may 
not apply to western populations. Second, the cut-off value of the AAPR needs external and prospective validation. Third, it is not clear whether the dynamic changes of the AAPR can reflect the prognosis and guide the treatment options.

Our current study confirmed the AAPR as an independent prognostic factor in HCC patients treated with TACE. Both AAPR-TNM and AAPR-CLIP outperformed their corresponding primary system in predicting OS. The prognostic value of the AAPR should be further validated in a larger prospective and multi-centre study.

\section{Abbreviations}

HCC: hepatocellular carcinoma; AFP: alpha fetoprotein; BUN: blood urea nitrogen; ALB: albumin; TBIL: total bilirubin; ALT: alanine aminotransferase; AST: aspartate aminotransferase; ALP: alkaline phosphatase; AAPR: albumin-to-alkaline phosphatase ratio; PVTT: portal vein tumour thrombus; ROC: receiver operating characteristic curve; AUC: area under curve; LRT: likelihood ratio test; TNM: Tumour-node-metastasis; NCCN: The National Comprehensive Cancer Network; TACE: transcatheter arterial chemoembolization. AJCC: The American Joint Committee on Cancer; CLIP: Cancer of the Liver Italian Program.

\section{Supplementary Material}

Supplementary tables.

http://www.jcancer.org/v09p3467s1.pdf

\section{Acknowledgements}

This study was supported by grants from the National Natural Science Foundation of China (grant no. 81502271, 31600710 and 81372374), the Combination Project of Production, the Scientific Research Project for University belonging to Guangzhou Municipal (grant no. 1201630019), the Natural Science Foundation of Guangdong (grant no. 2017A030313537, 2016A030313302, 2014A030312015 and 2014A030313146), the Project on the Integration of Industry, Education and Research of Guangdong Province (grant no. 2012B091100460), the Science Technology Program (grant no. 201709010030) and the Medical Scientific Research Foundation of Guangdong Province (grant no. A2015109).

We thank Hong-wei Guo for helping us with follow-up of patients.

\section{Competing Interests}

The authors have declared that no competing interest exists.

\section{References}

1. Chen W, Zheng R, Baade PD, Zhang S, Zeng H, Bray F, et al. Cancer statistics in China, 2015. CA: a cancer journal for clinicians. 2016; 66: 115-32.

2. Siegel RL, Miller KD, Jemal A. Cancer statistics, 2018. CA: a cancer journal for clinicians. 2018; 68: 7-30.

3. Bruix J, Gores GJ, Mazzaferro V. Hepatocellular carcinoma: clinical frontiers and perspectives. Gut. 2014; 63: 844-55.

4. Geschwind JF, Gholam PM, Goldenberg A, Mantry P, Martin RC, Piperdi B, et al. Use of Transarterial Chemoembolization (TACE) and Sorafenib in Patients with Unresectable Hepatocellular Carcinoma: US Regional Analysis of the GIDEON Registry. Liver Cancer. 2016; 5: 37-46.

5. Killock D. Immunotherapy: Nivolumab keeps HCC in check and opens avenues for checkmate. Nature reviews Clinical oncology. 2017; 14: 392.

6. Llovet JM, Ricci S, Mazzaferro V, Hilgard P, Gane E, Blanc JF, et al. Sorafenib in advanced hepatocellular carcinoma. The New England journal of medicine. 2008; 359: 378-90.

7. Cheng AL, Kang YK, Chen Z, Tsao CJ, Qin S, Kim JS, et al. Efficacy and safety of sorafenib in patients in the Asia-Pacific region with advanced hepatocellular carcinoma: a phase III randomised, double-blind, placebo-controlled trial. The Lancet Oncology. 2009; 10: 25-34.

8. Tsurusaki M, Murakami T. Surgical and Locoregional Therapy of HCC: TACE. Liver Cancer. 2015; 4: 165-75.

9. Kim JM, Kwon CH, Joh JW, Park JB, Ko JS, Lee JH, et al. The effect of alkaline phosphatase and intrahepatic metastases in large hepatocellular carcinoma. World J Surg Oncol. 2013; 11: 40.

10. Xu XS, Wan Y, Song SD, Chen W, Miao RC, Zhou YY, et al. Model based on gamma-glutamyltransferase and alkaline phosphatase for hepatocellular carcinoma prognosis. World Journal of Gastroenterology : WJG. 2014; 20: 10944-52.

11. Chan AW, Chan SL, Mo FK, Wong GL, Wong VW, Cheung YS, et al. Albumin-to-alkaline phosphatase ratio: a novel prognostic index for hepatocellular carcinoma. Disease markers. 2015; 2015: 564057.

12. Shahid M, Mubeen A, Tse J, Kakar S, Bateman AC, Borger D, et al. Branched chain in situ hybridization for albumin as a marker of hepatocellular differentiation: evaluation of manual and automated in situ hybridization platforms. The American journal of surgical pathology. 2015; 39: 25-34.

13. Toyoda H, Kumada T, Tada T, Sone Y, Kaneoka Y, Maeda A. Tumor Markers for Hepatocellular Carcinoma: Simple and Significant Predictors of Outcome in Patients with HCC. Liver Cancer. 2015; 4: 126-36.

14. Nie M, Sun P, Chen C, Bi X, Wang Y, Yang H, et al. Albumin-to-Alkaline Phosphatase Ratio: A Novel Prognostic Index of Overall Survival in Cisplatin-based Chemotherapy-treated Patients with Metastatic Nasopharyngeal Carcinoma. J Cancer. 2017; 8: 809-15.

15. Cai X-R, Chen Z-H, Chen J, Ma X-K, Bai M-J, Wang T-T, et al. Albumin-to-Alkaline Phosphatase Ratio as an Independent Prognostic Factor for Overall Survival of Advanced Hepatocellular Carcinoma Patients without Receiving Standard Anti-Cancer Therapies. J Cancer. 2018; 9: 189-97.

16. Tang A, Cruite I, Mitchell DG, Sirlin CB. Hepatocellular carcinoma imaging systems: why they exist, how they have evolved, and how they differ. Abdominal radiology. 2018; 43: 3-12.

17. Harimoto N, Yoshizumi T, Sakata K, Nagatsu A, Motomura T, Itoh S, et al. Prognostic significance of combined albumin-bilirubin and tumor-node-metastasis staging system in patients who underwent hepatic resection for hepatocellular carcinoma. Hepatology research : the official journal of the Japan Society of Hepatology. 2017; 47 (12): 1289-1298

18. Chan AW, Chong CC, Mo FK, Wong J, Yeo W, Johnson PJ, et al. Incorporating albumin-bilirubin grade into the cancer of the liver Italian program system for hepatocellular carcinoma. Journal of gastroenterology and hepatology. 2017; 32: 221-8.

19. Ji F, Liang Y, Fu SJ, Guo ZY, Shu M, Shen SL, et al. A novel and accurate predictor of survival for patients with hepatocellular carcinoma after surgical resection: the neutrophil to lymphocyte ratio (NLR) combined with the aspartate aminotransferase/platelet count ratio index (APRI). BMC cancer. 2016; 16: 137.

20. Chen ZH, Qiu MZ, Wu XY, Wu QN, Lu JH, Zeng ZL, et al. Elevated baseline serum lactate dehydrogenase indicates a poor prognosis in primary duodenum adenocarcinoma patients. J Cancer. 2018; 9: 512-20.

21. Arroyo V, Garcia-Martinez R, Salvatella X. Human serum albumin, systemic inflammation, and cirrhosis. Journal of hepatology. 2014; 61: 396-407.

22. Nojiri S, Joh T. Albumin suppresses human hepatocellular carcinoma proliferation and the cell cycle. International Journal of Molecular Sciences. 2014; 15: 5163-74.

23. Matsumoto H, Erickson RH, Gum JR, Yoshioka M, Gum E, Kim YS. Biosynthesis of alkaline phosphatase during differentiation of the human colon cancer cell line Caco-2. Gastroenterology. 1990; 98: 1199-207.

24. Ali NN, Rowe J, Teich NM. Constitutive expression of non-bone/liver/kidney alkaline phosphatase in human osteosarcoma cell lines. Journal of bone and mineral research : the official journal of the American Society for Bone and Mineral Research. 1996; 11: 512-20.

25. Sharma U, Pal D, Prasad R. A novel role of alkaline phosphatase in the ERK1/2 dephosphorylation in renal cell carcinoma cell lines: a new plausible therapeutic target. Biochimie. 2014; 107 Pt B: 406-9. 
26. Chen ZH, Hong YF, Lin J, Li X, Wu DH, Wen JY, et al. Validation and ranking of seven staging systems of hepatocellular carcinoma. Oncol Lett. 2017; 14: 705-14.

27. Chen $\mathrm{ZH}$, Hong $\mathrm{YF}$, Chen $\mathrm{X}$, Chen J, Lin $\mathrm{Q}$, Lin J, et al. Comparison of five staging systems in predicting the survival rate of patients with hepatocellular carcinoma undergoing trans-arterial chemoembolization therapy. Oncol Lett. 2018; 15: 855-62. 